\title{
Separation of variables in the Jacobi identities
}

\author{
Benito Hernández-Bermejo ${ }^{1} \quad$ Víctor Fairén \\ Departamento de Fúsica Matemática y Fluidos, Universidad Nacional de Educación a Distan- \\ cia. Senda del Rey S/N, 28040 Madrid, Spain.
}

\begin{abstract}
A new family of $n$-dimensional solutions of the Jacobi identities is characterized. Such a family is very general, thus unifying in a common framework many different well-known Poisson systems seemingly unrelated. This unification is not only conceptual, but also allows the development of general global methods of analysis.
\end{abstract}

Keywords: Finite-dimensional Poisson systems - Jacobi identities — Casimir invariants — Darboux reduction - PDEs.

${ }^{1}$ Corresponding author. E-mail: bhernand@apphys.uned.es 


\section{Introduction}

Poisson structures [1]-[3] have an important presence in all fields of Mathematical Physics, such as dynamical systems theory [4]-[10], fluid dynamics [11], magnetohydrodynamics [12], optics [13], continuous media [14], etc. Describing a given physical system in terms of a Poisson structure opens the possibility of obtaining a wide range of information which may be in the form of perturbative solutions [15], invariants [9, 16], nonlinear stability analysis [17], bifurcation properties and characterization of chaotic behaviour [13], or integrability results [18], to cite a few.

Mathematically, a finite-dimensional dynamical system is said to have a Poisson structure if it can be written in terms of a set of ODEs of the form:

$$
\dot{x}^{i}=\sum_{j=1}^{n} J^{i j} \partial_{j} H, \quad i=1, \ldots, n,
$$

where $H(\mathbf{x})$, which is usually taken to be a time-independent first integral, plays the role of Hamiltonian function. The $J^{i j}(\mathbf{x})$ are the entries of an $n \times n$ matrix $\mathcal{J}$ which may be degenerate in rank - known as the structure matrix - and they have the property of being solutions of the Jacobi identities:

$$
\sum_{l=1}^{n}\left(J^{l i} \partial_{l} J^{j k}+J^{l j} \partial_{l} J^{k i}+J^{l k} \partial_{l} J^{i j}\right)=0
$$

In (2), $\partial_{l}$ means $\partial / \partial x^{l}$ and indices $i, j, k$ run from 1 to $n$. The $J^{i j}$ must also verify the additional condition of being skew-symmetric:

$$
J^{i j}=-J^{j i} \quad \text { for all } i, j
$$

The possibility of describing a given finite-dimensional dynamical system in terms of a Poisson structure is still an open problem $[4,6,7,8,10,19,20]$. The source of the difficulty arises not only from the need of a known first integral playing the role of the Hamiltonian, but mainly due to the necessity of associating a suitable structure matrix to the problem. In other words, finding an appropriate solution of the Jacobi identities (2), complying also with the additional conditions (3), is unavoidable. This explains, together with the intrinsic mathematical interest of the problem, the permanent attention deserved in the literature by the obtainment and classification of skew-symmetric solutions of the Jacobi identities. In the simplest case of three-dimensional flows it has been possible to rewrite equations (2-3) in more manageable forms allowing the determination of some families of solutions [6, 7]. However, this strategy is not applicable when analyzing the general $n$-dimensional problem (2-3). In such a case, the present-day classification of solutions of (2-3) can be summarized, roughly speaking, as a sequence of families of solutions having increasing nonlinearity: constant structures (of which the well-known symplectic matrix is just a particular case), linear (i.e. Lie-Poisson) structures [21], affine-linear structures [22] and finally quadratic structures [8, 10, 23, 24].

In this letter we present a new family of skew-symmetric, separable solutions of the Jacobi identities. Due to the unusual generality of such a family -it consists of $n$-dimensional 
solutions not limited to a given degree of nonlinearity - many known Poisson structures appear embraced as particular cases, thus unifying many different systems seemingly unrelated. As we shall see, this unification has relevant consequences for the analysis of such systems, since it leads to the development of a common framework for the determination of many important properties such as the symplectic structure or the Darboux canonical form. Such properties can now be characterized globally in a unified and very economic way.

The structure of the article is as follows: In Section 2 we establish the main results concerning the form of the solutions and their properties. In Section 3 we illustrate the previous results by means of a number of examples, chosen to show the generality of the solutions and how very different systems, in principle unrelated, appear now grouped naturally and can be analyzed in a unified manner. We conclude in Section 4 with some final remarks.

\section{Poisson structures arising from separation of variables in the Jacobi identities}

Let $\left\{\varphi^{1}\left(x^{1}\right), \varphi^{2}\left(x^{2}\right), \ldots, \varphi^{n}\left(x^{n}\right)\right\}$ be a set of nonvanishing $C^{1}$ functions defined on a subset $\Omega \subset \mathbb{R}^{n}$. The need for the nonvanishing condition $\varphi^{i}\left(x^{i}\right) \neq 0$ in $\Omega$ will become clear in what

follows. In the context of this article we define a separable matrix as an $n \times n$ matrix defined in $\Omega$ which is of the form:

$$
S^{i j}=a^{i j} \varphi^{i}\left(x^{i}\right) \varphi^{j}\left(x^{j}\right), \quad a^{i j} \in \mathbb{R}, \quad a^{i j}=-a^{j i} \text { for all } i, j
$$

Obviously, every separable matrix thus defined is skew-symmetric. Moreover, every separable matrix is also a solution of the Jacobi identities (2). Therefore, every separable matrix is a structure matrix.

To prove this, we only need to substitute matrix $S^{i j}(4)$ into the Jacobi equations (2). We first arrive to terms of the form:

$$
S^{l i} \partial_{l} S^{j k}=a^{l i} a^{j k} \varphi^{l} \varphi^{i}\left(\delta^{l j} \dot{\varphi}^{j} \varphi^{k}+\delta^{l k} \varphi^{j} \dot{\varphi}^{k}\right)
$$

where $\dot{\varphi}^{i}$ means $\mathrm{d} \varphi^{i} / \mathrm{d} x^{i}$ and $\delta^{i j}$ is Kronecker's delta. Similar expressions are found for the other combinations of indexes. Grouping into (2) and simplifying the deltas we arrive at:

$$
\begin{gathered}
\sum_{l=1}^{n}\left(S^{l i} \partial_{l} S^{j k}+S^{l j} \partial_{l} S^{k i}+S^{l k} \partial_{l} S^{i j}\right)= \\
\varphi^{i} \varphi^{j} \varphi^{k}\left[\dot{\varphi}^{j}\left(a^{j i} a^{j k}+a^{i j} a^{j k}\right)+\dot{\varphi}^{k}\left(a^{j k} a^{k i}+a^{k j} a^{k i}\right)+\dot{\varphi}^{i}\left(a^{k i} a^{i j}+a^{i k} a^{i j}\right)\right]=0
\end{gathered}
$$

due to the skew-symmetry of the $a^{i j}$. This demonstrates one of the main results of this paper.

Therefore, in what follows we shall analyze the main properties of the separable structure matrices. According to the previous result, these are all of the form:

$$
J^{i j}=a^{i j} \varphi^{i}\left(x^{i}\right) \varphi^{j}\left(x^{j}\right), \quad a^{i j} \in \mathbb{R}, \quad a^{i j}=-a^{j i} \text { for all } i, j
$$

where the $\varphi^{i}$ are nonvanishing arbitrary $C^{1}$ functions.

We shall start by considering the determination of the Casimir invariants. This can be done with only the knowledge of the structure matrix, since they are Hamiltonian independent. 
In order to solve this problem, the most efficient possibility is to use the abbreviated method developed by Hernández-Bermejo and Fairén [9]. For this, we first notice that $\operatorname{Rank}(\mathcal{J})=$ $\operatorname{Rank}(A)$ in $\Omega$, where $(A)^{i j}=a^{i j}$. This is a consequence of the nonvanishing character of the $\varphi^{i}$ in $\Omega$. According to [9], this associates naturally the Casimir invariants to the kernel of matrix $A$ and leads to the determination of their form: If

$$
C=\sum_{j=1}^{n} k^{j} \int \frac{\mathrm{d} x^{j}}{\varphi^{j}\left(x^{j}\right)}, \quad \mathbf{k}=\left(k^{1}, k^{2}, \ldots, k^{n}\right)^{T} \in \operatorname{Ker}(A)
$$

where the superscript ${ }^{T}$ denotes the transpose of a matrix, then $C$ is a Casimir function of $\mathcal{J}$. In addition, we have that $\operatorname{dim}\{\operatorname{Ker}(A)\}=n-\operatorname{Rank}(A) \equiv m$, and thus there are $m$ linearly independent vectors that span this kernel. Let $\left\{\mathbf{k}_{(1)}, \mathbf{k}_{(2)}, \ldots, \mathbf{k}_{(m)}\right\}$ be a basis of $\operatorname{Ker}(A)$, and let

$$
C_{(i)}=\sum_{j=1}^{n} k_{(i)}^{j} \int \frac{\mathrm{d} x^{j}}{\varphi^{j}\left(x^{j}\right)}, \quad i=1,2, \ldots, m
$$

be the $m$ Casimir invariants associated to this basis. Then, if we evaluate the Jacobian matrix associated with $\left\{C_{(1)}, C_{(2)}, \ldots, C_{(m)}\right\}$ it can be immediately seen that such Casimir invariants are functionally independent. Since $m$ is also the corank of $\mathcal{J}$, this demonstrates that $\left\{C_{(1)}, C_{(2)}, \ldots, C_{(m)}\right\}$ is a complete set of independent Casimir functions of the structure matrix (7), any other Casimir invariant being a functional combination of them. Therefore, the symplectic foliation of the separable structure matrices can be completely determined from the kernel of the constant matrix $A$, which is a significant simplification of the problem.

We now examine the reduction to the Darboux canonical form. For this, we shall take into account the equation for the transformation of the structure matrix under general diffeomorphisms, $y^{i}=y^{i}(x)$ :

$$
\tilde{J}^{i j}(y)=\sum_{k, l=1}^{n} \frac{\partial y^{i}}{\partial x^{k}} J^{k l}(x) \frac{\partial y^{j}}{\partial x^{l}}
$$

We thus introduce the following diffeomorphic transformation, which is globally defined in $\Omega$ :

$$
y^{i}=\int \frac{\mathrm{d} x^{i}}{\varphi^{i}\left(x^{i}\right)}, \quad i=1, \ldots, n
$$

When (7) and (11) are substituted in (10), we obtain:

$$
\tilde{J}^{i j}(y)=a^{i j}, \quad \text { for all } i, j
$$

In other words, we have transformed the matrix in such a way that now $\tilde{\mathcal{J}}=A$ is a structure matrix of constant entries. In addition to this, we now perform a second transformation, which is also globally defined:

$$
z^{i}=\sum_{j=1}^{n} P^{i j} y^{j}, \quad i=1, \ldots, n
$$

where $P$ is a constant, $n \times n$ invertible matrix. According to (10), the structure matrix $\tilde{J}$ now becomes:

$$
\hat{\mathcal{J}}=P \cdot \tilde{\mathcal{J}} \cdot P^{T}=P \cdot A \cdot P^{T}
$$


It is well known [25] that matrix $P$ in (14) can be chosen to have:

$$
\hat{\mathcal{J}}=\operatorname{diag}(D_{1}, D_{2}, \ldots, D_{r / 2}, \overbrace{0, \ldots, 0}^{(n-r)})
$$

where $r=\operatorname{Rank}(A)$ is an even number because $A$ is skew-symmetric, and

$$
D_{1}=D_{2}=\ldots=D_{r / 2}=\left(\begin{array}{cc}
0 & 1 \\
-1 & 0
\end{array}\right)
$$

With (15-16), the structure matrix has been reduced to the Darboux form, since $\hat{\mathcal{J}}$ is a direct sum of $2 \times 2$ symplectic matrices plus $(n-r)$ null $1 \times 1$ matrices corresponding to the Casimir invariants, which in the Darboux representation are decoupled and correspond to the variables $\left\{z_{r+1}, \ldots, z_{n}\right\}$. It is worth emphasizing that the reduction has been completed explicitly and globally on the domain of interest. This is interesting, since the number of Poisson structures for which this can be done is exceedingly limited. Well on the contrary, in the present case this is possible in a quite natural way.

In addition to these advantageous manipulation properties, the separable structure matrices embrace and unify many different Poisson structures common in the literature. We shall now see a sample in the following section.

\section{Examples}

(I) Lotka-Volterra and Generalized Lotka-Volterra systems

The following kind of separable structure matrices

$$
J^{i j}=a^{i j} x^{i} x^{j}, \quad a^{i j} \in \mathbb{R}, \quad a^{i j}=-a^{j i} \text { for all } i, j
$$

were first recognized by Plank [8] in the characterization of Poisson structures of the LotkaVolterra equations, and were important later in the wider case of generalized Lotka-Volterra Poisson systems [10]. However, particular cases of (17) had been previously found in different contexts, such as plasma physics [26] or population dynamics $[4,6,27]$ (see also the relativistic Toda equations in the next example).

In (17) we have $\varphi^{i}\left(x^{i}\right)=x^{i}$, and therefore the Casimir invariants are immediately found to be, according to (8), of the form:

$$
C=\sum_{j=1}^{n} k^{j} \ln \left(x^{j}\right), \quad \mathbf{k}=\left(k^{1}, k^{2}, \ldots, k^{n}\right)^{T} \in \operatorname{Ker}(A)
$$

In the specific case of Lotka-Volterra equations, the first integrals (18) were already noticed by Volterra himself [28], but they were not generically recognized as Casimir invariants until Plank's work [8]. Being Hamiltonian independent, they also appear in more general types of models sharing the structure matrix (17), such as those treated in [10].

The first transformation (11) necessary to achieve the Darboux canonical form now is:

$$
y^{i}=\int \frac{\mathrm{d} x^{i}}{\varphi^{i}\left(x^{i}\right)}=\ln \left(x^{i}\right), \quad i=1, \ldots, n
$$


The change of variables (19) is to be followed by the linear transformation (13). This kind of two-step reduction to a classical Hamiltonian formulation has been known for long —outside the framework of Poisson structures - in the particular case of conservative, even-dimensional Lotka-Volterra systems [29]. The realization that such reduction is, in fact, Hamiltonian independent and inherent to structure matrices of the kind (17) was formalized recently in [10].

\section{(II) Toda lattice and relativistic Toda lattice}

Toda system, when expressed in Flaschka's variables [30] $\left(\alpha^{1}, \ldots, \alpha^{N-1}, \beta^{1}, \ldots, \beta^{N}\right)$ is a Poisson system with brackets $\left\{\alpha^{i}, \beta^{i}\right\}=-\alpha^{i},\left\{\alpha^{i}, \beta^{i+1}\right\}=\alpha^{i}$, while the rest of the elementary brackets vanish. This Poisson bracket corresponds to a separable structure given by:

$$
\begin{gathered}
\varphi^{i}\left(\alpha^{i}\right)=\alpha^{i}, \quad i=1, \ldots, N-1 \\
\varphi^{j}\left(\beta^{j}\right)=1, \quad j=1, \ldots, N \\
A=\left(\begin{array}{cc}
O_{(N-1) \times(N-1)} & M_{(N-1) \times N} \\
-M_{N \times(N-1)}^{T} & O_{N \times N}
\end{array}\right)
\end{gathered}
$$

where the subindexes of the submatrices indicate their sizes, $O$ denotes the null matrix and

$$
M_{(N-1) \times N}=\left(\begin{array}{cccccc}
-1 & 1 & 0 & \ldots & 0 & 0 \\
0 & -1 & 1 & \ldots & 0 & 0 \\
\vdots & \vdots & \vdots & & \vdots & \vdots \\
0 & 0 & 0 & \ldots & -1 & 1
\end{array}\right)
$$

It is immediate that the kernel of $A$ is one-dimensional, a basis of which is provided by the vector $\mathbf{k}=(\overbrace{0, \ldots, 0}^{N-1}, \overbrace{1, \ldots, 1}^{N})^{T}$. Consequently, from (8) there is only one independent Casimir invariant, $C=\sum_{j=1}^{N} \int \mathrm{d} \beta^{j}=\sum_{j=1}^{N} \beta^{j}$, which is the result found in [30]. The reduction to the Darboux form also becomes straightforward, since we have to perform transformation (11)

$$
\begin{aligned}
& \tilde{\alpha}^{i}=\ln \left(\alpha^{i}\right), \quad i=1, \ldots, N-1 \\
& \tilde{\beta}^{j}=\beta^{j}, \quad j=1, \ldots, N
\end{aligned}
$$

and then carry out the linear change of variables (13).

Analogously, we consider now the relativistic Toda equations expressed in similar variables [30] $\left(\alpha^{1}, \ldots, \alpha^{N-1}, \beta^{1}, \ldots, \beta^{N}\right)$. Again, it is a Poisson system with brackets $\left\{\alpha^{i}, \alpha^{i+1}\right\}=$ $\alpha^{i} \alpha^{i+1},\left\{\alpha^{i}, \beta^{i}\right\}=-\alpha^{i} \beta^{i},\left\{\alpha^{i}, \beta^{i+1}\right\}=\alpha^{i} \beta^{i+1}$, while the rest of the elementary brackets vanish. This Poisson bracket corresponds to a separable structure of the form (17) examined in Example I. Therefore, all the considerations made there hold in this context.

(III) Further examples: Constant structure matrices, Kermack-McKendric model, circle maps, $2 \times 2$ games

We end the present section with a brief enumeration of other examples which have also deserved some interest in the literature. We shall not elaborate on them with the detail of the previous instances, but only outline the most interesting features. 
The first example will be that of the constant skew-symmetric structure matrices, which appear frequently in very diverse developments. In this case we would like to recall that $\varphi^{i}\left(x^{i}\right)=1$, and thus the Casimir functions (8) are linear. Notice also that transformation (11) reduces to the identity, and therefore the reduction to classical Hamiltonian form only involves a linear transformation (13-15). These are well-known facts that now appear as a simple particular case.

As a second example we touch upon the Kermack-McKendric model [5, 6], which admits a Poisson structure in terms of matrix:

$$
\mathcal{J}=\left(\begin{array}{ccc}
0 & -r x^{1} x^{2} & 0 \\
r x^{1} x^{2} & 0 & -a x^{2} \\
0 & a x^{2} & 0
\end{array}\right)
$$

where the $x^{i}$ denote the system variables and $\{a, r\}$ are real constants. We again have a separable matrix with $\left\{\varphi^{1}\left(x^{1}\right), \varphi^{2}\left(x^{2}\right), \varphi^{3}\left(x^{3}\right)\right\}=\left\{x^{1}, x^{2}, 1\right\}$. Therefore, this example turns out to be very similar to the nonrelativistic Toda lattice examined before, as it can be seen from (20-21). We thus find that seemingly unrelated problems can be analyzed in a similar, unifying framework.

Next we shall mention the Poisson structures appearing in the study of certain circle maps [6], in which we have:

$$
\mathcal{J}=\left(\begin{array}{ccc}
0 & 0 & -\left(x^{1}\right)^{2}\left(x^{3}\right)^{2} \\
0 & 0 & -\left(x^{2}\right)^{2}\left(x^{3}\right)^{2} \\
\left(x^{1}\right)^{2}\left(x^{3}\right)^{2} & \left(x^{2}\right)^{2}\left(x^{3}\right)^{2} & 0
\end{array}\right)
$$

We thus have $\varphi^{i}\left(x^{i}\right)=\left(x^{i}\right)^{2}$, a more nonlinear kind of function. The evaluation of the Casimir invariants and the Darboux canonical form do not present any special difficulty in this case and are omitted.

Finally we shall consider a very different kind of structure matrix found in the context of $2 \times 2$ games [31], in which:

$$
\mathcal{J}=\left(\begin{array}{cc}
0 & x^{1}\left(1-x^{1}\right) x^{2}\left(1-x^{2}\right) \\
-x^{1}\left(1-x^{1}\right) x^{2}\left(1-x^{2}\right) & 0
\end{array}\right)
$$

Now we have that $\Omega=\operatorname{int}\left\{S_{1} \times S_{1}\right\}$ is the interior of the product of two probability simplices, and $\varphi^{i}\left(x^{i}\right)=x^{i}\left(1-x^{i}\right)$. Obviously there are no Casimir functions in this case. Because now $A$ is the $2 \times 2$ symplectic matrix, no linear transformation (13) is required and the reduction to the classical Hamiltonian form only involves transformation (11):

$$
y^{i}=\int \frac{\mathrm{d} x^{i}}{x^{i}\left(1-x^{i}\right)}=\ln \left(\frac{x^{i}}{1-x^{i}}\right), \quad i=1,2
$$

Notice that all the manipulations are properly defined because functions $\varphi^{i}\left(x^{i}\right)$ are nonvanishing in the domain $\Omega$. 


\section{Final remarks}

In this letter we have presented a new family of skew-symmetric solutions of the Jacobi identities which exhibits a number of interesting properties:

- It can be found by applying the classical method of separation of variables. It is mathematically remarkable that such a technique, in principle restricted to the domain of certain linear PDEs, can be applied in this context.

- The resulting solutions are valid for the $n$-dimensional problem (2-3), independently of the value of $n$, and are not limited to a given degree of nonlinearity. Well on the contrary, they can contain polynomials of arbitrary degree and general functions as well.

- They generalize already known solutions. In fact, they unify in a common framework many different Poisson structures and well-known systems which seemed to be unrelated, and now appear as particular cases of a common family. We have provided a list of them in the examples.

And last but not least:

- This unification is not only conceptual, but allows the development of general methods of analysis simultaneously valid for every particular case. Specifically, it is possible to determine in an algorithmic and explicit way the Casimir invariants and the Darboux canonical form. Moreover, these results hold globally in the phase-space - in contrast with the usual scope of Darboux' theorem [3], which only guarantees a local reduction.

As we have seen, the direct search of new Poisson structures as solutions of the Jacobi identities leads to a unifying perspective of very diverse, and seemingly unrelated, systems. This is useful not only from a classification point of view - from which we have obtained a remarkably general family of solutions - but also because it is possible, as we have demonstrated, to develop a unified approach for their analysis which becomes much more economic and elegant than a heuristic, case-by-case strategy. Obviously, it is feasible to establish such techniques with regard to all Hamiltonian independent properties, such as those treated above.

For these reasons, it is the authors' impression that this kind of analysis of the Jacobi equations can produce interesting results for further generalization and unification of finitedimensional Poisson structures. In particular, two clear lines of investigation are an additional generalization of the separable solutions (7) found in this paper, as well as the search of completely different families of structure matrices. These possibilities will be the subject of future research.

\section{Acknowledgements}

The authors wish to acknowledge support from the European Union (Esprit WG 24490). 


\section{References}

[1] A. Lichnerowicz, J. Diff. Geom. 12 (1977) 253.

[2] A. Weinstein, J. Diff. Geom. 18 (1983) 523.

[3] P. J. Olver, Applications of Lie Groups to Differential Equations, 2nd Ed. (SpringerVerlag, New York, 1993).

[4] Y. Nutku, Phys. Lett. A 145 (1990) 27.

[5] Y. Nutku, J. Phys. A: Math. Gen. 23 (1990) L1145.

[6] H. Gümral and Y. Nutku, J. Math. Phys. 34 (1993) 5691.

[7] F. Haas and J. Goedert, Phys. Lett. A 199 (1995) 173.

[8] M. Plank, J. Math. Phys. 36 (1995) 3520.

[9] B. Hernández-Bermejo and V. Fairén, Phys. Lett. A 241 (1998) 148.

[10] B. Hernández-Bermejo and V. Fairén, J. Math. Phys. 39 (1998) 6162.

[11] P. J. Morrison, Rev. Mod. Phys. 70 (1998) 467.

[12] P. J. Morrison and J. M. Greene, Phys. Rev. Lett. 45 (1980) 790.

[13] D. David, D. D. Holm and M. V. Tratnik, Phys. Rep. 187 (1990) 281.

[14] I. E. Dzyaloshinskii and G. E. Volovick, Ann. Physics 125 (1980) 67.

[15] J. R. Cary and R. G. Littlejohn, Ann. Physics 151 (1983) 1.

[16] T. W. Yudichak, B. Hernández-Bermejo and P. J. Morrison, Phys. Lett. A 260 (1999) 475 .

[17] D. D. Holm, J. E. Marsden, T. Ratiu and A. Weinstein, Phys. Rep. 123 (1985) 1.

[18] F. Magri, J. Math. Phys. 19 (1978) 1156.

[19] V. Perlick, J. Math. Phys. 33 (1992) 599.

[20] V. V. Kozlov, Symmetries, Topology and Resonances in Hamiltonian Mechanics (Springer Verlag, New York, 1996).

[21] S. Lie, Theorie der Transformationsgruppen (B. G. Teubner, Leipzig, 1888).

[22] K. H. Bhaskara, Proc. Indian Acad. Sci. Math. Sci. 100 (1990) 189.

[23] K. H. Bhaskara and K. Rama, J. Math. Phys. 32 (1991) 2319.

[24] Z.-J. Liu and P. Xu, Lett. Math. Phys. 26 (1992) 33. 
[25] F. Ayres Jr., Schaum's Outline of Matrices (McGraw-Hill, New York, 1962).

[26] G. Picard and T. W. Johnston, Phys. Rev. Lett. 48 (1982) 1610.

[27] L. Cairó and M. R. Feix, J. Phys. A: Math. Gen. 25 (1992) L1287.

[28] V. Volterra, Leçons sur la Théorie Mathématique de la Lutte pour la Vie (Gauthier Villars, Paris, 1931).

[29] E. H. Kerner, Phys. Lett. A 151 (1990) 401.

[30] P. A. Damianou, J. Math. Phys. 35 (1994) 5511.

[31] J. Hofbauer, J. Math. Biol. 34 (1996) 675. 